PROCEEDINGS OF THE

AMERICAN MATHEMATICAL SOCIETY

Volume 133, Number 12, Pages 3535-3542

S 0002-9939(05)07892-5

Article electronically published on June 6, 2005

\title{
BOUNDEDNESS OF OPERATORS ON HARDY SPACES VIA ATOMIC DECOMPOSITIONS
}

\author{
MARCIN BOWNIK
}

(Communicated by Andreas Seeger)

\begin{abstract}
An example of a linear functional defined on a dense subspace of the Hardy space $H^{1}\left(\mathbb{R}^{n}\right)$ is constructed. It is shown that despite the fact that this functional is uniformly bounded on all atoms, it does not extend to a bounded functional on the whole $H^{1}$. Therefore, this shows that in general it is not enough to verify that an operator or a functional is bounded on atoms to conclude that it extends boundedly to the whole space. The construction is based on the fact due to Y. Meyer which states that quasi-norms corresponding to finite and infinite atomic decompositions in $H^{p}, 0<p \leq 1$, are not equivalent.
\end{abstract}

\section{INTRODUCTION}

The intended purpose of this work is not only of research, but also of pedagogical nature, since it is based on an already published, but quite possibly not well-known, example of Y. Meyer.

In this note we give a rather surprising example of a linear functional defined on a dense subspace of $H^{1}$, which maps all atoms into bounded scalars, but yet it cannot be extended to a bounded functional on the whole space $H^{1}$. As a consequence of this example, it follows that in general it does not suffice to check that an operator from a Hardy space $H^{p}, 0<p \leq 1$, into some other quasi-Banach space $X$ maps atoms into bounded elements of $X$ to verify that this operator extends to a bounded operator on $H^{p}$. An untrained reader might inadvertently draw such a conclusion by reading literature on atomic decompositions of Hardy spaces. Here we list a few references, which could potentially lead someone into this not fully justified belief [3. Proof of Lemma II.2], [5, Corollary 6.3], 8, Lemma 5.1], 10, Chapter 6.7.c], [14, Chapter 6.3], 16. Chapter III.3.3], 17, Chapter 1], and [18, Proposition 6.13].

Despite this, it is important to emphasize that to verify boundedness for many important classes of operators defined on $H^{p}$ spaces, it is indeed sufficient to check that atoms are mapped into bounded elements of $X$. Probably the best known example of a class with this property are Calderón-Zygmund operators. The complete proof of this fact (based on atomic decomposition of $H^{p}$ spaces) can be found, for

Received by the editors July 8, 2004.

2000 Mathematics Subject Classification. Primary 42B30.

The author was partially supported by NSF grant DMS-0441817.

(C)2005 American Mathematical Society Reverts to public domain 28 years from publication 
example, in [9, Chapter III.7] or [10, Theorem 6.7.1] for convolution-type CalderónZygmund operators and in [1, Chapter 1.9] or [15, Lemma 1 in Chapter 7.3] for non-convolution operators.

A rudimentary set of facts about real-variable theory of Hardy spaces can be found in [4, 7, 8, 9, 10, 16. Here, we limit ourselves to the basic definition of real-variable $H^{p}$ spaces due to Fefferman and Stein [7].

Definition 1. We say that a tempered distribution $f \in \mathcal{S}^{\prime}\left(\mathbb{R}^{n}\right)$ belongs to the Hardy space $H^{p}\left(\mathbb{R}^{n}\right), 0<p<\infty$, if its radial maximal function $M_{\varphi}^{0} f$ (or equivalently non-tangential maximal function $\left.M_{\varphi} f\right)$ is in $L^{p}$. Here, $\varphi$ is any test function in the Schwartz class $\mathcal{S}\left(\mathbb{R}^{n}\right)$ with $\int \varphi \neq 0$, and

$$
\begin{aligned}
& M_{\varphi}^{0} f(x)=\sup _{t>0}\left|f * \varphi_{t}(x)\right|, \\
& M_{\varphi} f(x)=\sup _{t>0} \sup _{|y-x|<t}\left|f * \varphi_{t}(y)\right|,
\end{aligned}
$$

where $\varphi_{t}(x)=t^{-n} \varphi(x / t)$.

A fundamental result of Fefferman and Stein asserts that this definition does not depend on the choice of $\varphi \in \mathcal{S}$ (as long as $\int \varphi \neq 0$ ) and $H^{p}\left(\mathbb{R}^{n}\right)$ with the quasi-norm $\|f\|_{H^{p}}=\left\|M_{\varphi}^{0} f\right\|_{L^{p}}$ (or $\|f\|_{H^{p}}=\left\|M_{\varphi} f\right\|_{L^{p}}$ ) is a quasi-Banach space. Moreover, $H^{p}\left(\mathbb{R}^{n}\right)=L^{p}\left(\mathbb{R}^{n}\right)$ for $p>1$. For proofs of these facts we refer to [16, Chapter III.1].

\section{EXAmple of Meyer}

In this section we present an example of an atom in $H^{p}$ whose norm is not achieved by its finite atomic decomposition. The first example of this kind for $H^{1}$ was exhibited by Y. Meyer [13; see also [9, Section III.8.3]. Here, we merely adapt this example to a more general $H^{p}\left(\mathbb{R}^{n}\right), 0<p \leq 1$, case.

We start by recalling a definition of an atom for $H^{p}$ spaces. For the sake of simplicity, we will use only $L^{\infty}$ normalization for our atoms and we will limit ourselves to the classical isotropic Hardy spaces $H^{p}\left(\mathbb{R}^{n}\right)$ given by Definition 1 .

Definition 2. We say that a function $a$ is a $p$-atom, where $0<p \leq 1$, if

$$
\begin{aligned}
\operatorname{supp} a & \subset B\left(x_{0}, r\right) \quad \text { for some } x_{0} \in \mathbb{R}^{n}, r>0, \\
\|a\|_{\infty} & \leq\left|B\left(x_{0}, r\right)\right|^{-1 / p}, \\
\int_{\mathbb{R}^{n}} a(x) x^{\alpha} d x & =0 \quad \text { for all }|\alpha| \leq\lfloor(1 / p-1) n\rfloor .
\end{aligned}
$$

Here, $B\left(x_{0}, r\right)=\left\{x \in \mathbb{R}^{n}:\left|x-x_{0}\right|<r\right\}$.

Let $\Theta^{k}\left(\mathbb{R}^{n}\right)$ be the space of all (finite) linear combinations of $p$-atoms, that is,

$$
\Theta^{k}\left(\mathbb{R}^{n}\right)=\left\{f \in L^{\infty}\left(\mathbb{R}^{n}\right): \operatorname{supp} f \text { is bounded and } \int_{\mathbb{R}^{n}} f(x) x^{\alpha} d x=0 \text { for }|\alpha| \leq k\right\} .
$$

It is well known that $\Theta^{k}\left(\mathbb{R}^{n}\right)$ is a dense subspace of $H^{p}\left(\mathbb{R}^{n}\right), 0<p \leq 1$, for sufficiently large $k$, that is, for $k \geq\lfloor(1 / p-1) n\rfloor$. In fact, an even smaller space $\Theta^{k}\left(\mathbb{R}^{n}\right) \cap C^{\infty}\left(\mathbb{R}^{n}\right)$ is also a dense subspace of $H^{p}\left(\mathbb{R}^{n}\right)$ for the same range of $k$ 's. 
On the space $\Theta^{k}\left(\mathbb{R}^{n}\right)$ we consider two quasi-norms corresponding to finite and infinite atomic decompositions:

$$
\begin{aligned}
& \|f\|_{H^{p}, \infty}=\inf \left\{\left(\sum_{i=1}^{\infty}\left|\lambda_{i}\right|^{p}\right)^{1 / p}: f=\sum_{i=1}^{\infty} \lambda_{i} a_{i}, \quad a_{i} \text { is a } p \text {-atom for } i \in \mathbb{N}\right\} . \\
& \|f\|_{H^{p},<\infty}=\inf \left\{\left(\sum_{i=1}^{N}\left|\lambda_{i}\right|^{p}\right)^{1 / p}: f=\sum_{i=1}^{N} \lambda_{i} a_{i},\right.
\end{aligned}
$$$$
\left.a_{i} \text { is a } p \text {-atom for } 1 \leq i \leq N \text {, and } N \in \mathbb{N}\right\} .
$$

It should be emphasized that the equality $f=\sum_{i=1}^{\infty} \lambda_{i} a_{i}$ in (6) is understood in the sense of tempered distributions $\mathcal{S}^{\prime}\left(\mathbb{R}^{n}\right)$. This follows from a standard $H^{p}$ theory fact stating that for any choice of coefficients $\left(\lambda_{i}\right)_{i=1}^{\infty} \in \ell^{p}(\mathbb{N})$ and $p$-atoms $a_{i}$ 's, the series $\sum_{i=1}^{\infty} \lambda_{i} a_{i}$ converges in $\|\cdot\|_{H^{p}}$ quasi-norm, and hence in $\mathcal{S}^{\prime}$.

The atomic decomposition theorem of Coifman [2] for $H^{p}$ spaces states that the converse is also true, i.e., every element $f \in H^{p}\left(\mathbb{R}^{n}\right)$ can be decomposed as $f=\sum_{i=1}^{\infty} \lambda_{i} a_{i}$ for some choice of $\lambda_{i}$ 's and $p$-atoms $a_{i}$ 's. Moreover,

$$
\|f\|_{H^{p}} \asymp\|f\|_{H^{p}, \infty} \quad \text { for all } f \in H^{p},
$$

and hence for all $f \in \Theta^{k}\left(\mathbb{R}^{n}\right)$, where $k \geq\lfloor(1 / p-1) n\rfloor$.

A less-known result due to Y. Meyer states that the above is not true when the quasi-norm $\|\cdot\|_{H^{p}, \infty}$ is replaced by $\|\cdot\|_{H^{p},<\infty}$. Hence, the quasi-norms $\|\cdot\|_{H^{p}, \infty}$ and $\|\cdot\|_{H^{p},<\infty}$ are not equivalent on $\Theta^{k}\left(\mathbb{R}^{n}\right)$.

Theorem 1. Suppose $0<p \leq 1$ and $k \geq\lfloor(1 / p-1) n\rfloor$. Then for arbitrarily small $\varepsilon>0$, there exists $f \in \Theta^{k}\left(\mathbb{R}^{n}\right)$ such that

$$
\|f\|_{H^{p}, \infty}<\varepsilon \quad \text { and } \quad\|f\|_{H^{p},<\infty}=1 .
$$

Proof. Let $a$ be a $p$-atom supported on the unit ball $B(0,1)$ with

$$
\int_{\mathbb{R}^{n}} a(x) x^{\alpha} d x=0 \quad \text { for all }|\alpha| \leq k,
$$

and such that

$$
|a(x)| \geq c|B(0,1)|^{-1 / p}>0 \quad \text { for a.e. } x \in B(0,1) .
$$

To show that an atom $a$ satisfying (10) exists, let $K=\sum_{i=0}^{k}\left(\begin{array}{c}n-1+i \\ i\end{array}\right)$ be the cardinality of the collection of all multi-indices $\alpha$ with $|\alpha| \leq k$. Then, we claim that it suffices to construct a finite partition $\left\{E_{i}\right\}_{i=1}^{m}$ of $B(0,1)$ such that the vectors

$$
v_{i}=\left(\int_{E_{i}} x^{\alpha} d x\right)_{|\alpha| \leq k} \in \mathbb{R}^{K}, \quad i=1, \ldots, m,
$$

span the whole space $\mathbb{R}^{K}$ even if one of them is removed, i.e.,

$$
\forall 1 \leq i_{0} \leq m \quad \operatorname{span}\left\{v_{i}: 1 \leq i \leq m, i \neq i_{0}\right\}=\mathbb{R}^{K} .
$$

Indeed, (12) implies that there exist non-zero coefficients $c_{1}, \ldots, c_{m}$ such that $\sum_{i=1}^{m} c_{i} v_{i}=0$. Moreover, by scaling we may also assume that $\sup _{1 \leq i \leq m}\left|c_{i}\right| \leq$ $|B(0,1)|^{-1 / p}$. Then, one can immediately verify that

$$
a(x)=\sum_{i=1}^{m} c_{i} \mathbf{1}_{E_{i}}(x)
$$


is a required atom satisfying (10) with $c=|B(0,1)|^{1 / p} \inf _{1 \leq i \leq m}\left|c_{i}\right|$. Finally, we remark that a partition $\left\{E_{i}\right\}_{i=1}^{m}$ satisfying (11) and (12) can easily be found by an inductive partitioning of the ball.

Next, we choose a collection of pairwise disjoint balls $\left\{B_{i}\right\}_{i \in \mathbb{N}}$ such that $B_{i} \subset$ $B(0,1)$ for all $i \in \mathbb{N}$,

$$
U:=\bigcup_{i \in \mathbb{N}} B_{i} \text { is dense in } B(0,1) \quad \text { and } \quad|U|=\sum_{i \in \mathbb{N}}\left|B_{i}\right|<c \varepsilon^{p} .
$$

For each $i \in \mathbb{N}$, let $a_{i}$ be a dilated and translated copy of the atom $a$ with support adjusted to the ball $B_{i}$. That is, if $B_{i}=B\left(x_{0}, r\right)$, then $a_{i}(x)=r^{-n / p} a\left(\left(x-x_{0}\right) / r\right)$. As a consequence of (10) each $a_{i}$ is an atom supported on $B_{i}$ and satisfying

$$
\left|a_{i}(x)\right| \geq c\left|B_{i}\right|^{-1 / p} \quad \text { for a.e. } x \in B_{i} .
$$

Let

$$
f(x)=c^{-1 / p} \sum_{i \in \mathbb{N}}\left|B_{i}\right|^{1 / p} a_{i}(x) .
$$

Then, it is obvious that $\|f\|_{H^{p}, \infty}^{p} \leq \sum_{i \in \mathbb{N}}\left|B_{i}\right| / c<\varepsilon^{p}$. On the other hand, we claim that $\|f\|_{H^{p}, \infty}$ must remain large.

Indeed, suppose that $f$ has a finite atomic decomposition $f=\sum_{i=1}^{N} \lambda_{i} b_{i}$, where each $b_{i}$ is supported on a ball $\tilde{B}_{i}$. Let $g$ be a majorant of $f$ given by

$$
g=\left(\sum_{i=1}^{N}\left|\lambda_{i}\right|^{p}\left|\tilde{B}_{i}\right|^{-1} \mathbf{1}_{\tilde{B}_{i}}\right)^{1 / p} .
$$

By (13) and (14)

$$
\begin{aligned}
\mathbf{1}_{U}(x) \leq|f(x)| \leq \sum_{i=1}^{N}\left|\lambda_{i}\right|\left|b_{i}(x)\right| \leq & \left(\sum_{i=1}^{N}\left|\lambda_{i}\right|^{p}\left|b_{i}(x)\right|^{p}\right)^{1 / p} \\
& \leq\left(\sum_{i=1}^{N}\left|\lambda_{i}\right|^{p}\left|\tilde{B}_{i}\right|^{-1} \mathbf{1}_{\tilde{B}_{i}}(x)\right)^{1 / p}=g(x) .
\end{aligned}
$$

Since $g$ is continuous everywhere almost everywhere (possibly with the exception of the union of boundaries of a finite collection of balls $\left.\bigcup_{i=1}^{N} \partial\left(\tilde{B}_{i}\right)\right)$ and $U$ is dense in $B(0,1)$, hence $g(x) \geq 1$ for a.e. $x \in B(0,1)$. Therefore,

$$
|B(0,1)| \leq \int_{B(0,1)} g(x)^{p} d x=\sum_{i=1}^{N}\left|\lambda_{i}\right|^{p} .
$$

Consequently, $\|f\|_{H^{p},<\infty} \geq|B(0,1)|^{1 / p}$. It is also immediate from (14) that $\|f\|_{H^{p},<\infty} \leq c^{-1 / p}|B(0,1)|^{1 / p}$. Since $\varepsilon>0$ was arbitrary, by a simple rescaling we find $f$ satisfying (8), which completes the proof of Theorem 1 .

It is perhaps worthwhile to recall the original example of Meyer, which through its simplicity better illustrates the idea of the above proof; see also [9, Chapter III.8].

Example 1. For arbitrarily small $\varepsilon>0$, we will construct a function $f \in \Theta^{0}(\mathbb{R})$ such that

$$
\|f\|_{H^{1}, \infty}<\varepsilon \quad \text { and } \quad\|f\|_{H^{1},<\infty}=1 .
$$


Let $\left\{B_{i}\right\}_{i \in \mathbb{N}}$ be a collection of pairwise disjoint intervals $\subset[0,1]$ such that $U:=$ $\bigcup_{i \in \mathbb{N}} B_{i}$ is dense in $[0,1]$ and $|U|<\varepsilon$. Let $a_{i}$ be a 1-atom supported on $B_{i}$, which equals $1 /\left|B_{i}\right|$ on the left half of $B_{i}$ and $-1 /\left|B_{i}\right|$ on the other half. Let $f(x)=\sum_{i \in \mathbb{N}}\left|B_{i}\right| a_{i}(x)$. It is clear that $\|f\|_{H^{1}, \infty} \leq \sum_{i \in \mathbb{N}}\left|B_{i}\right|<\varepsilon$ and $|f(x)|=1$ for a.e. $x \in U$.

To see that $\|f\|_{H^{1},<\infty}=1$, consider a finite atomic decomposition $f=\sum_{i=1}^{N} \lambda_{i} b_{i}$, where each $b_{i}$ is supported on the interval $\tilde{B}_{i}$. Then

$$
\mathbf{1}_{U}(x)=|f(x)| \leq \sum_{i=1}^{N}\left|\lambda_{i}\right|\left|a_{i}(x)\right| \leq \sum_{i=1}^{N}\left|\lambda_{i}\right|\left|\tilde{B}_{i}\right|^{-1} \mathbf{1}_{\tilde{B}_{i}}(x)=: g(x) .
$$

Since $g$ is discontinuous only on a finite number of points and $U \subset[0,1]$ is dense, hence $g(x) \geq 1$ for a.e. $x \in[0,1]$. Integrating $g(x)$ over $[0,1]$ yields $\|f\|_{H^{1},<\infty} \geq 1$. Since $f$ is itself a 1 -atom supported on $[0,1]$, hence $\|f\|_{H^{1},<\infty}=1$.

\section{UNBOUNDED LINEAR FUNCTIONALS ON $H^{1}$}

The goal of this section is to show the existence of a linear functional on a dense subspace of $H^{1}$, which does not extend to a bounded functional on the whole $H^{1}$ despite the fact that it maps all 1-atoms into scalars with universally bounded absolute values. The existence of such a functional will follow from Meyer's example and an application of the Hahn-Banach Theorem.

Theorem 2. There exists a linear functional l on $\Theta^{0}\left(\mathbb{R}^{n}\right)$ such that

$$
|l(f)| \leq\|f\|_{H^{1},<\infty} \quad \text { for all } f \in \Theta^{0}\left(\mathbb{R}^{n}\right),
$$

which does not extend to a bounded functional on $H^{1}\left(\mathbb{R}^{n}\right)$, i.e.,

$$
\sup _{f \in \Theta^{0}\left(\mathbb{R}^{n}\right)}|l(f)| /\|f\|_{H^{1}, \infty}=\infty .
$$

In particular, $l$ is uniformly bounded on all atoms in $H^{1}\left(\mathbb{R}^{n}\right)$. That is, $|l(a)| \leq 1$ for every 1-atom a.

Proof. Suppose $\left\{x_{i}\right\}_{i \in \mathbb{N}} \subset \mathbb{R}^{n}$ is any sequence such that $B\left(x_{i}, 1\right) \cap B\left(x_{j}, 1\right)=\emptyset$ for every $i \neq j$. For each $i \in \mathbb{N}$, let $a_{i}(x)$ be a function in $\Theta^{0}\left(\mathbb{R}^{n}\right)$ supported on the ball $B\left(x_{i}, 1\right)$ and satisfying

$$
\left\|a_{i}\right\|_{H^{1}, \infty}<1 / i \quad \text { and } \quad\left\|a_{i}\right\|_{H^{1},<\infty}=1 .
$$

In addition, from the proof of Theorem 1 we can also assume that

$$
\left|a_{i}(x)\right| \geq c /|B(0,1)|>0 \quad \text { for } x \in U_{i}, \text { where } U_{i} \subset B\left(x_{i}, 1\right) \text { is dense. }
$$

Here, $c$ is a constant independent of $i \in \mathbb{N}$. In fact, we can choose $a_{i}$ 's such that $c=1$ is the largest possible by taking atoms taking only two non-zero and opposite values as in Example 1.

Let $V=\operatorname{span}\left\{a_{i}(x): i \in \mathbb{N}\right\} \subset \Theta^{0}\left(\mathbb{R}^{n}\right)$ be the space of all finite linear combinations of the above functions. We claim that

$$
c \sum_{i \in \mathbb{N}}\left|c_{i}\right| \leq \|\left. f\right|_{H^{1},<\infty} \leq \sum_{i \in \mathbb{N}}\left|c_{i}\right| \quad \text { for all } f(x)=\sum_{i \in \mathbb{N}} c_{i} a_{i}(x) \in V .
$$

Therefore, $V$ is isomorphic to the subspace of $\ell^{1}(\mathbb{N})$ consisting of sequences with finite support. 
To show (20), we proceed as in the proof of Theorem 1 Suppose that $f(x)=$ $\sum_{i \in \mathbb{N}} c_{i} a_{i}(x) \in V$ has a finite atomic decomposition $f(x)=\sum_{j=1}^{N} \lambda_{j} b_{j}(x)$, where each $b_{j}$ is supported on a ball $B_{j}$. By (19)

$$
\frac{c}{|B(0,1)|} \sum_{i \in \mathbb{N}}\left|c_{i}\right| \mathbf{1}_{U_{i}}(x) \leq|f(x)| \leq \sum_{j=1}^{N}\left|\lambda_{j}\right|\left|b_{j}(x)\right| \leq \sum_{j=1}^{N}\left|\lambda_{j}\right|\left|B_{j}\right|^{-1} \mathbf{1}_{B_{j}}(x)=: g(x) .
$$

Since $g$ is continuous everywhere almost everywhere (possibly with the exception of the union of boundaries of a finite collection of balls $\left.\bigcup_{j=1}^{N} \partial\left(B_{j}\right)\right)$ and each $U_{i}$ is dense in $B\left(x_{i}, 1\right)$, hence

$$
g(x) \geq \frac{c}{|B(0,1)|} \sum_{i \in \mathbb{N}}\left|c_{i}\right| \mathbf{1}_{B\left(x_{i}, 1\right)}(x) \quad \text { for a.e. } x \in \mathbb{R}^{n} .
$$

Therefore,

$$
c \sum_{i \in \mathbb{N}}\left|c_{i}\right| \leq \int_{\mathbb{R}^{n}} g(x) d x=\sum_{j=1}^{N}\left|\lambda_{j}\right| .
$$

This shows the lower bound in (20). The upper bound in (20) is trivial by the triangle inequality and (18).

Define a linear functional $l$ initially on $V$ by

$$
l(f)=\sum_{i \in \mathbb{N}} c_{i} \quad \text { for } f(x)=\sum_{i \in \mathbb{N}} c_{i} a_{i}(x) \in V .
$$

By (20), $l$ is a bounded functional on a subspace of $V$ of a normed space $\Theta^{0}\left(\mathbb{R}^{n}\right)$ equipped with the norm $\|\cdot\|_{H^{1},<\infty}$. Moreover, the norm of $l$ is at most 1 . Therefore, by the Hahn-Banach Theorem, $l$ extends to a bounded functional on the whole space $\Theta^{0}\left(\mathbb{R}^{n}\right)$ such that (16) holds. Since, $l\left(a_{i}\right) /\left\|a_{i}\right\|_{H^{1}, \infty} \geq i$ and $i \in \mathbb{N}$ is arbitrary, we also have (17), which completes the proof of Theorem 2 .

Remark 1. We remark that the proof of Theorem 2 can be easily modified to show the existence of a linear functional $l$ defined on some subspace of $V \subset \Theta^{k}\left(\mathbb{R}^{n}\right)$, where $k \geq\lfloor(1 / p-1) n\rfloor, 0<p \leq 1$, which is bounded on $V$ equipped with the quasi-norm $\|\cdot\|_{H^{p},<\infty}$, but is unbounded as a functional on $V$ with the quasinorm $\|\cdot\|_{H^{p}, \infty}$. However, since the Hahn-Banach Theorem is not valid on general quasi-normed spaces, there is no guarantee that this functional can be boundedly extended to the whole $\Theta^{k}\left(\mathbb{R}^{n}\right)$.

In fact, Duren, Romberg, and Shields [6] characterized the duals of classical Hardy spaces on the unit complex disc $H^{p}(\mathbb{D})$ for $0<p<1$ and used it to show that the Hahn-Banach Theorem fails for these spaces. Furthermore, Kalton [11, 12] showed that a quasi-Banach space $X$ has the Hahn-Banach Extension property (continuous linear functionals on a closed subspace extend to the whole space) if and only if it is a Banach space.

Finally, we discuss how Theorem 2 relates to the problem of showing boundedness of operators on Hardy spaces via atomic decompositions. A typical argument invoked for that purpose is as follows.

Suppose $T$ is a linear operator defined on some dense subspace $D$ of $H^{p}\left(\mathbb{R}^{n}\right)$, $0<p \leq 1$, into some quasi-Banach space $X$, with the property that $\|T(a)\|_{X} \leq$ $C<\infty$ for all $p$-atoms $a$ and some universal constant $C$. Here, we implicitly require that $\Theta^{k}\left(\mathbb{R}^{n}\right) \subset D$, where $k=\lfloor(1 / p-1) n\rfloor$ and $\|\cdot\|_{X}$ satisfies $p$-triangle inequality 
$\|f+g\|_{X}^{p} \leq\|f\|_{X}^{p}+\|g\|_{X}^{p}$. To show that $T$ extends to a bounded operator from $H^{p}$ to $X$, consider an arbitrary element $f \in \Theta^{k}\left(\mathbb{R}^{n}\right)$. By the atomic decomposition theorem for $H^{p}$ spaces, we can represent $f=\sum_{i \in \mathbb{N}} \lambda_{i} a_{i}$, where the $a_{i}$ 's are $p$-atoms and $\sum_{i \in \mathbb{N}}\left|\lambda_{i}\right|^{p} \leq C_{0}\|f\|_{H^{p}}$ for some universal constant $C_{0}$. Since

$$
T f=T\left(\sum_{i \in \mathbb{N}} \lambda_{i} a_{i}\right)=\sum_{i \in \mathbb{N}} \lambda_{i} T\left(a_{i}\right),
$$

hence

$$
\|T f\|_{X}^{p} \leq \sum_{i \in \mathbb{N}}\left\|\lambda_{i} T\left(a_{i}\right)\right\|_{X}^{p} \leq C \sum_{i \in \mathbb{N}}\left|\lambda_{i}\right|^{p} \leq C C_{0}\|f\|_{H^{p}} .
$$

Since $f$ was arbitrary, (22) shows that $T$ extends to a bounded operator $T: H^{p} \rightarrow$ $X$.

The main problem with this argument is that in general there is no guarantee that (21) is valid due the fact that the sum in (21) is infinite. Theorem 2 shows that this is not only a theoretical possibility, but (21) may indeed fail in certain situations (at least when $p=1$ ).

The above argument also has a variant, where infinite atomic decomposition is replaced with a finite one $f=\sum_{i=1}^{N} \lambda_{i} a_{i}$, where the $a_{i}$ 's are $p$-atoms and $\sum_{i=1}^{N}\left|\lambda_{i}\right|^{p} \leq C_{0}\|f\|_{H^{p}}$ for some constant $C_{0}$. This time the problem lies with the fact that $C_{0}$ cannot be chosen universally for all $f \in \Theta^{k}\left(\mathbb{R}^{n}\right)$ as it is evidenced by Theorem 1 .

Therefore, in light of Theorems 1 and 2 we must undoubtedly admit that in general it is not enough to verify that an operator or a functional is merely bounded on $p$-atoms to conclude that it extends boundedly to the whole space $H^{p}, 0<p \leq 1$. It is also necessary to verify an identity such as (21), asserting that $T$ behaves well with respect to infinite atomic decompositions. This in turn is not always a trivial task, e.g. in the case of Calderón-Zygmund operators it requires use of certain approximation arguments. For further details, we refer to [1, 9, 15].

\section{REFERENCES}

1. M. Bownik, Anisotropic Hardy spaces and wavelets, Mem. Amer. Math. Soc. 164 (2003), no. 781 , vi+122 pp. MR.1982689 (2004e:42023)

2. R.R. Coifman, A real variable characterization of $H^{p}$, Studia Math. 51 (1974), 269-274. MR0358318 (50:10784)

3. R.R. Coifman, P.-L. Lions, Y. Meyer, and S. Semmes, Compensated compactness and Hardy spaces, J. Math. Pures Appl. 72 (1993), 247-286. MR1225511(95d:46033)

4. R.R. Coifman, G. Weiss, Extensions of Hardy spaces and their use in analysis, Bull. Amer. Math. Soc. 83 (1977), 569-645. MR0447954(56:6264)

5. J. Duoandikoetxea, Fourier Analysis, American Math. Soc., Providence (2001). MR1800316 (2001k:42001)

6. P.L. Duren, B.W. Romberg, and A.L. Shields, Linear functionals on $H^{p}$ spaces with $0<p<1$, J. Reine Angew. Math. 238 (1969), 32-60. MR0259579(41:4217)

7. C. Fefferman, E.M. Stein, $H^{p}$ spaces of several variables, Acta Math. 129 (1972), 137-193. MR 0447953 (56:6263)

8. G.B. Folland, E.M. Stein, Hardy spaces on homogeneous groups, Princeton University Press, Princeton, N.J., 1982. MR0657581 (84h:43027)

9. J. García-Cuerva, J.L. Rubio de Francia, Weighted Norm Inequalities and Related Topics, North-Holland (1985). MR0807149 (87d:42023)

10. L. Grafakos, Classical and Modern Fourier Analysis, Pearson (2004).

11. N.J. Kalton, Basic sequences in F-spaces and their applications, Proc. Edinburgh Math. Soc. 19 (1974/75), 151-167. MR0415259 (54:3350) 
12. N.J. Kalton, Quasi-Banach Spaces in "Handbook of geometry of Banach spaces, Vol. II", 1099-1130, North-Holland, Amsterdam (2003). MR 1999192 (2005a:46004)

13. Y. Meyer, M. Taibleson, and G. Weiss, Some functional analytic properties of the spaces $B_{q}$ generated by blocks, Indiana Univ. Math. J. 34 (1985), 493-515. MR0794574 (87c:46036)

14. Y. Meyer, Wavelets and operators, Cambridge University Press, Cambridge (1992). MR.1228209 (94f:42001)

15. Y. Meyer, R. Coifman, Wavelets. Calderón-Zygmund and multilinear operators, Cambridge University Press, Cambridge (1997). MR.1456993 (98e:42001)

16. E.M. Stein, Harmonic Analysis: Real-Variable Methods, Orthogonality, and Oscillatory Integrals, Princeton Univ. Press (1993). MR1232192 (95c:42002)

17. M.H. Taibleson and G. Weiss, The molecular characterization of certain Hardy spaces, Astérisque 77 (1980), 67-149. MR0604370 (83g:42012)

18. P. Wojtaszczyk, A mathematical introduction to wavelets, Cambridge University Press, Cambridge (1997). MR1436437 (98j:42025)

Department of Mathematics, University of Oregon, Eugene, Oregon 97403-1222

E-mail address: mbownik@uoregon.edu 